\title{
*Radiation Effects Studies at the Advanced Photon Source
}

\author{
M. Petra ${ }^{\dagger}$, P.K. Den Hartog, E.R. Moog, S. Sasaki, N. Sereno and I.B. \\ Vasserman \\ Advanced Photon Source, Argonne National Laboratory, Argonne, IL 60439 US
}

\begin{abstract}
At the Advanced Photon Source (APS) concern for radiation-induced demagnetization of the insertion devices (IDs) in the storage ring and in the free-electron laser has initiated systematic radiation effects studies towards the development of efficient techniques for ID protection. The studies include radiation dose monitoring, parametric study of the radiation-induced demagnetization, as well as, potentially, a dedicated radiation effects testbed at the APS providing GeV electron beams. Such studies could also be directly applicable to future generation facilities, such as the Linac Coherent Light Source (LCLS). Results and discussion of the radiation damage studies at APS are presented.
\end{abstract}

PACS codes: 42.88, 41.85.L, 75.50.W

Keywords: radiation effects, wiggler magnets, permanent magnets

\section{Introduction}

The insertion devices (IDs) at the Advanced Photon Source (APS) storage ring and the freeelectron laser (FEL) are subjected to a harsh radiation environment that can cause radiationinduced demagnetization. This radiation environment is primarily composed of a broad energy spectrum of gamma rays, electrons, neutrons and $\mathrm{x}$-rays [1].

\footnotetext{
* The submitted manuscript has been created by the University of Chicago as Operator of Argonne National Laboratory ("Argonne") under Contract No. W31-109-ENG-38 with the U.S. Department of Energy. The U.S. Government retains for itself, and others acting on its behalf, a paid-up, nonexclusive, irrevocable worldwide license in said article to reproduce, prepare derivative works, distribute copies to the public, and perform publicly and display publicly,
} by or on behalf of the Government.

$\dagger$ Corresponding author. Tel.: +1-630-252-1382; fax:+1-630252-9303.

E-mail address: petra@aps.anl.gov (M. Petra). 
Radiation damage in materials is in general a function of the type and energy of the radiation, the integrated dose and dose rate, the irradiation temperature, the impurity content of the material and the irradiation history.

To date, several radiation damage studies of permanent magnets have been performed under electron, proton, deuteron, gamma and neutron irradiations [2-7]. However, results from radiation damage studies are sometimes difficult to interpret because of differences that are known to affect the likelihood of damage, such as the irradiation temperature, the sample geometry, and the grade and manufacturer of the magnet material.

Radiation damage studies have shown that charged particles and neutrons are far more effective at producing damage than low energy photons. In addition, it has been shown that the larger the coercivity, the higher the radiation resistance of the magnet [8]. SmCo permanent magnets have also been found to be more radiation resistant than $\mathrm{NdFeB}$ magnets that could be attributed to their higher Curie temperature and their superior thermal properties.

To date, radiation-induced demagnetization has been observed in two IDs at ESRF caused by electron beam misteering onto the vacuum chamber wall of the ID [9-10].

Further study is needed in understanding the damage process so that efficient techniques can be developed for magnet protection.

\section{Radiation Effects Studies}

\subsection{Dosimetry}

To date, dose monitoring at the APS has been performed with thermoluminescent dosimeters and radiachromic films. Integrated doses on the IDs using these techniques have been published elsewhere [11-12]. In 2002 a new high-dose dosimetry technique has been added i.e., alanine electron paramagnetic resonance (EPR) dosimetry.

Alanine is an amino acid in which disrupted molecular bonding due to ionizing radiation gives rise to free radicals. The radical concentration is a function of the absorbed dose that is then analyzed by EPR. This technique exhibits equivalent response for photons and electrons and an energyindependent response for photon energies above approximately $100 \mathrm{keV}$; however, dose underestimation occurs for photon energies below that value [13]. Absorbed doses up to $20 \mathrm{Mrad}$ can be measured with this technique.

Systematic dose monitoring using this technique has been initiated on the IDs in the APS storage ring and the FEL. Other critical components around the accelerator (such as CCD cameras, encoders, motors and cables) are also being monitored. Integrated doses on these components provide information on their lifetime in the APS radiation environment.

To date, dose monitoring on the IDs has been performed on the upstream (US) and the downstream (DS) ends of the devices. The dosimeters are being replaced at the end of each run (i.e., approximately in three-month time intervals). The US doses are being affected by the gap-size history during ID operation and the presence or absence of shielding upstream of the IDs; a number of IDs in the storage ring are not shielded due to space constraints.

To date, the highest recorded doses (i.e., of at least 10 Mrad during one run) have been observed during the 2001 runs in sector 3 of the APS storage ring. These doses are primarily due to increased beam losses caused by a recent reduction in the size of the ID vacuum chamber in that sector, that is, from an $8 \mathrm{~mm}$ aperture to a $5 \mathrm{~mm}$ aperture. The 5-mm aperture ID vacuum chamber is the limiting aperture in the storage ring and the first such chamber after the injection point is located in sector 3 . In addition, the APS storage ring was run in top-up mode as well as in a low emittance lattice, and the injection efficiency has not been optimized for these new conditions.

Absorbed doses in the other sectors around the storage ring have been systematically lower than those in sector 3 (i.e., of the order of $2 \mathrm{Mrad}$ or less during one run in several IDs). 
2.2 Radiation-Induced Damage to IDs at the APS Storage Ring

The IDs APS27\#2 and U27\#12 that are located in sector 3 suffered severe radiation damage that was more pronounced for $\mathrm{U} 27 \# 12$.

Abnormally high doses recorded by radiation dosimeters on those two devices during the run 2001-3 prompted removal of the devices from the storage ring and investigation for potential radiation damage. The device U27\#12 was removed on December 2001 for repair and reinserted in the storage ring (Fig. 1). At the end of the next run, it was again removed and checked for potential radiation damage, and further damage was found (Fig. 2).

The most severe radiation effects have been observed in sector 3; however, radiation damage has also been found in U33\#15 located in sector 1 .

Table 1 shows the rms phase errors measured for each of the demagnetized IDs initially, after the damage was observed, and after the ID was retuned. The amount of the mechanical taper introduced is also shown in the table. The third harmonic of the undulator radiation as calculated from the measured magnetic field is given in table 1 as a percentage of the ideal intensity that would be obtained from a perfect undulator. (The original undulator specification aimed at having the thirdharmonic intensity be at least $70 \%$ of the ideal.) The radiation damage had a particularly strong effect on the intensity of the third harmonic. Magnetic tuning was not able to remove all the damage from the downstream undulator. Instead, more extensive repair to this ID will be needed.
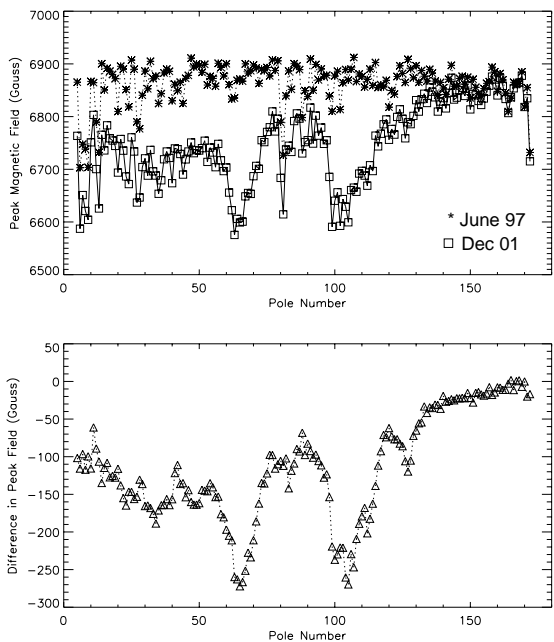

Fig. 1. Magnetic field changes in the downstream undulator in sector 3, U27\#12, at $10.5 \mathrm{~mm}$ gap. The top panel shows the peak magnetic field under each pole as measured in June 1997 (before the device was installed) and in Dec. 2001. The bottom panel shows the difference in the magnetic field. (The data for the weaker end poles are omitted.)

Extra shielding is being added in sector 3. In addition, temperature monitoring along the length of the IDs that suffered radiation damage has been initiated. Dose monitoring is also being performed along the length of the damaged IDs to allow comparisons between the absorbed dose and the radiation-induced demagnetization profiles.
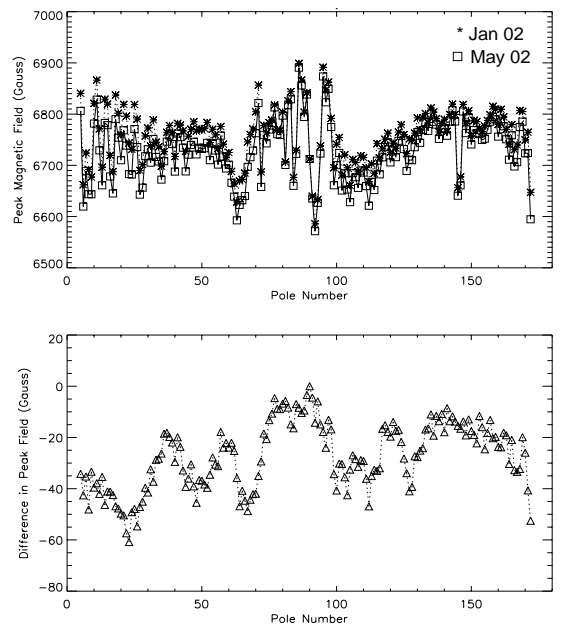

Fig. 2. Magnetic field changes in U27\#12 at $10.5 \mathrm{~mm}$ gap. The top panel shows the peak magnetic field under each pole as 
measured in Jan. 2002, after the device had been retuned, and in May 2002. The bottom panel shows the difference in the magnetic field. (The data for the weaker end poles are omitted.) The damage is less than that found previously, but clearly damage is continuing.

Table 1. rms phase errors for the demagnetized undulators initially, after observed damage, and after retuning.

\begin{tabular}{|c|c|c|c|}
\hline $\mathrm{U} 27 \# 12$ & \multicolumn{2}{|c|}{ Gap $10.5 \mathrm{~mm}$} & Sector 3 DS \\
\hline Date & $\begin{array}{l}\text { RMS } \\
\text { Phase } \\
\text { error }\end{array}$ & $\begin{array}{l}3^{\text {rd }} \\
\text { harm., } \% \\
\text { of ideal }\end{array}$ & comment \\
\hline 1997 June 23 & 5.45 & 82.6 & reference \\
\hline 2001 Dec. 31 & 36.5 & 35.2 & damaged \\
\hline 2002 Jan. 3 & 9.29 & 69.0 & $\begin{array}{l}\text { tuned, taper } \\
0.160 \mathrm{~mm}\end{array}$ \\
\hline 2002 May 6 & 14.14 & 52 & more damage \\
\hline 2002 May 7 & 10.81 & 62.4 & $\begin{array}{l}\text { tuned, taper } \\
0.025 \mathrm{~mm}\end{array}$ \\
\hline
\end{tabular}

APS27\#2
\begin{tabular}{|l|l|l|l|} 
Gap 11.5m & Sector 3 US \\
\hline 2000 June 23 & 2.62 & 91.5 & reference \\
\hline 2002 Jan. 8 & 10.79 & 64.2 & damaged \\
\hline 2002 Jan. 8 & 3.67 & 86.1 & $\begin{array}{c}\text { tuned, taper } \\
0.150 \mathrm{~mm}\end{array}$ \\
\hline
\end{tabular}

\begin{tabular}{|c|c|c|l|} 
U33\#15 & \multicolumn{2}{c}{ Gap $11.5 \mathrm{~mm}$} & Sector 1 DS \\
\hline 1997 Sept. 9 & 2.88 & 89.8 & reference \\
\hline 2002 May 2 & 5.91 & 82 & some damage \\
\hline 2002 May 3 & 5.14 & 84 & $\begin{array}{l}\text { tuned, taper } \\
0.040 \mathrm{~mm}\end{array}$ \\
\hline
\end{tabular}

Several of the permanent magnets used at the APS undulators are Shin Etsu N38H NdFeB magnets while those used for the LCLS prototype are Shin Etsu N39SH NdFeB magnets. Both types of magnets will be investigated for radiation sensitivity in future experiments. In addition, neutron activation analysis (NAA) is being performed in an LCLS sample magnet for impurity identification.

\subsection{Proposed Radiation-Damage Testbed at the APS}

A dedicated radiation-damage testbed for systematic study of radiation damage in permanent magnets is proposed for the APS. Potential use of the testbed for radiation-damage studies of materials other than magnets is also being investigated.

The proposed testbed will be capable of delivering $325 \mathrm{MeV}-7 \mathrm{GeV}$ electron beams at a maximum current of $5 \mathrm{nC} /$ per shot and a cycle rate of $2 \mathrm{~Hz}$. For that purpose, an existing stub line on the booster ring can be built out with a few new components (Fig. 3).

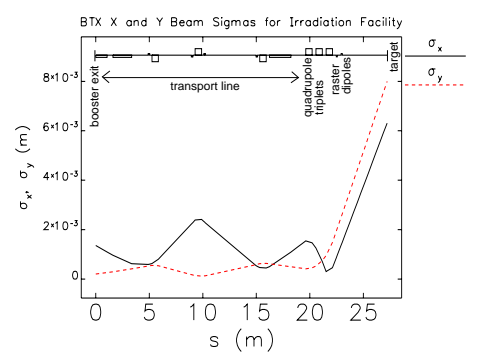

Fig. 3. Proposed radiation-damage testbed beamline and associated beam sigmas.

\section{Summary}

The radiation-induced demagnetization of IDs is a critical issue. Radiation damage has now been observed in a few IDs in the APS storage ring. Systematic dose monitoring provides the dosedistribution profiles around the APS storage ring and the FEL and identification of regions where the probability for damage is highest. A parametric study of the radiation-induced demagnetization process has been initiated.

\section{Acknowledgements}

The authors wish to thank Dr. Marc Desrosiers (National Institute of Standards and Technology) for helpful discussions on the alanine EPR dosimetry. This work was supported by the U.S. Department of Energy, Office of Science, BES, under contract No. W-31-109-ENG-38. NAA was performed at the University of Wisconsin-Madison nuclear reactor facility under the US DOE reactor sharing program.

\section{References}

[1] N. Ipe et al., Argonne National Laboratory 
report no. ANL/APS/TB-7 (1993).

[2] A.F. Zeller and J.A. Nolen, Proc. $9^{\text {th }}$ International Workshop on Rare Earth Magnets and their Applications, Bad Soden, FRG (1987) 157.

[3] T. Bizen et al., Nucl. Instrum. Methods A 467468 (2001) 185.

[4] O.P. Kahkonen et al., Europhys. Lett. 12(5) (1990) 413.

[5] S. Okuda, K. Ohashi and N. Kobayashi, Nucl. Instrum. Methods B 94 (1994) 227.

[6] J.R. Cost et al., IEEE Trans. Magn., 24 (1988) 2016.

[7] H.B. Luna et al., Nucl. Instrum. Methods A 285 (1989) 349.

[8] A.F. Zeller, "Radiation Damage in Permanent Magnetic Materials," National Superconducting Cyclotron Laboratory report (1999).

[9] J. Pflüger et al., Rev. Sci. Instrum. 66(2) (1995) 1946.

[10] J. Chavanne et al., ESRF Machine Technical Note 1-1996/ID (1996).

[11] E.R. Moog et al., Proc. Synchrotron Radiation Instrumentation: Tenth US National Conference (1997) 219.

[12] J. Alderman et al., Argonne National Laboratory report no. LS-283 (2000).

[13]E.L. Florian et al., CERN report no. CERN/TIS-CFM/IR/93-03 (1993). 